\title{
Simulating the In-Field AC and DC Performance of High-Temperature Superconducting Coils
}

\author{
Mark D. Ainslie, Member, IEEE, Di Hu, Jin Zou, and David A. Cardwell
}

\begin{abstract}
In this paper, the authors investigate numerically the in-field behaviour of high-temperature superconducting (HTS) coils and a method to potentially improve their performance using ferromagnetic material as a flux diverter. The ability to accurately predict the electromagnetic behaviour of superconductors in complex geometries and electromagnetic environments is crucial to the design of commercially-viable superconductor-based electrical devices, such as power transmission cables, superconducting fault current limiters, transformers, and motors and generators. The analysis is carried out using a two-dimensional (2D) axisymmetric model of a circular pancake coil based on the $H$-formulation and implemented in Comsol Multiphysics 4.3a. We explore the use of flux diverters to improve an HTS coil's performance with respect to its DC (maximum allowable/critical current) and AC (AC loss) characteristics, for various background magnetic fields. It is found that while flux diverters can improve the AC properties of coils, they can be detrimental to the DC properties in this particular configuration.
\end{abstract}

Index Terms-AC loss reduction, high-temperature superconductors (HTSs), superconducting coils, transport ac loss.

\section{INTRODUCTION}

TN THIS PAPER, the authors investigate numerically the in$\mathbf{I}_{\text {field behaviour of high-temperature superconducting (HTS) }}$ coils and a method to potentially improve their performance using ferromagnetic material as a flux diverter. The ability to accurately predict the electromagnetic behaviour of superconductors in complex geometries is crucial to the design of commercially-viable superconductor-based electrical devices, such as power transmission cables, superconducting fault current limiters, transformers, and motors and generators. In a superconducting electric machine, in particular, the superconducting coils operate in a rather complex electromagnetic environment [1], [2]. This also allows investigation of the performance of coils acting as HTS insert coils in high-field magnets [3], for example.

The analysis is carried out using a two-dimensional (2D) axisymmetric model of a circular pancake coil based on the $\boldsymbol{H}$ formulation and implemented in the commercial software

Manuscript received August 10, 2014. M. D. Ainslie would like to acknowledge financial support from a Royal Academy of Engineering Research Fellowship. D. Hu and J. Zou would like to acknowledge financial support from Churchill College, Cambridge, the China Scholarship Council and the Cambridge Commonwealth, European and International Trust.

M. D. Ainslie, D. Hu, J. Zou and D. A. Cardwell are with the Bulk Superconductivity Group, Department of Engineering, University of Cambridge, Cambridge CB2 1PZ, UK (e-mail: mark.ainslie@eng.cam.ac.uk, dh455@cam.ac.uk, jz351@cam.ac.uk, dc135@cam.ac.uk). package Comsol Multiphysics. Research carried out to date on the electromagnetic properties of superconductors operating within complex geometries such as coils has produced a number of interesting results; in particular, how the use of hybrid combinations of ferromagnetic materials and superconductors can improve the electromagnetic properties of the latter [5]. Specifically, we explore the use of flux diverters to improve an HTS coil's performance with respect to its DC (maximum allowable current) and AC (AC loss) characteristics, for various DC background magnetic fields. Previous work, such as [2], [4], has investigated the effect on the AC loss of coils subjected to AC magnetic fields, including AC ripple fields superimposed on a DC background field, whilst carrying a DC transport current. This analysis acts to provide a foundation for determining the performance of such coils in-field in working superconducting devices.

\section{Modelling FramewORK}

Figure 1 shows the 2D axisymmetric model of a circular pancake coil of $N$ turns used in this paper. The model consists of an array of $N$ superconducting tapes representing the crosssection of the coil, symmetric around $r=0$, with an inner radius $r_{\mathrm{i}}$ and outer radius $r_{\mathrm{o}}$. The superconducting layers are surrounded by an air sub-domain.

The finite-element model is based on the $\boldsymbol{H}$-formulation, which directly solves the magnetic field components $\boldsymbol{H}=\left[H_{\mathrm{r}}\right.$, $H_{z}$ ] derived from Ampere's and Faraday's Laws, and is implemented here in 2D axisymmetric form using the AC/DC module of Comsol Multiphysics 4.3a [6]. More detailed information about the model formulation can be found in [7], [8], which was extended in [5], [9], [10] to allow for the inclusion of magnetic materials.

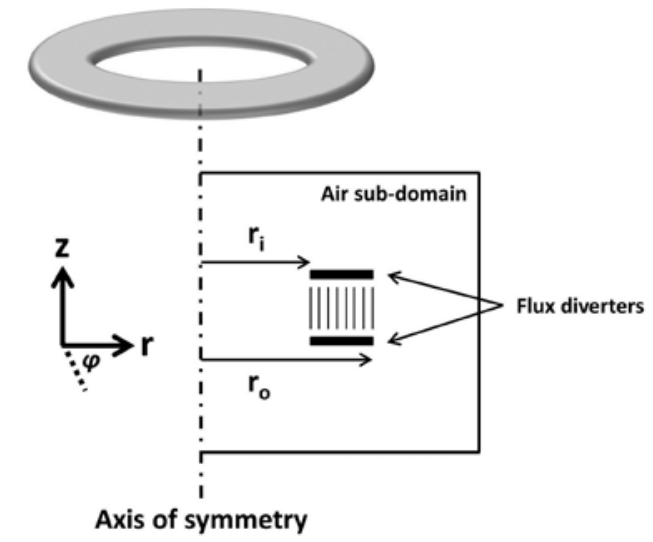

Fig. 1. Two-dimensional (2D) axisymmetric model of a superconducting coil used as a basis for this analysis. 
The electrical behavior of the superconductor is modelled by an $E-J$ power law relation [11], [12], $E \alpha J^{\mathrm{n}}$, where $n=20$. The in-field dependence of the superconductor's critical current density, $J_{\mathrm{c}}(B)$, is modelled (to simplify the comparison between models) using an isotropic relationship based on a generalized Kim dependence [13]:

$$
J_{\mathrm{c}}(B)=J_{\mathrm{c} 0} /\left(1+B / B_{0}\right)^{\beta}
$$

The parameters for (1) are calculated based on an average of the measured $J_{\mathrm{c}}(B, \theta)$ data (not yet published) between 0 $0.7 \mathrm{~T}$ for a short sample of SCS4050 2G HTS wire (manufactured by SuperPower, Inc. [14]) at $77 \mathrm{~K}$, for which $J_{\mathrm{c} 0}=2.5 \times 10^{10} \mathrm{~A} / \mathrm{m}^{2}, B_{0}=0.13$ and $\beta=0.743$. Other properties of the superconducting tape under analysis are listed in Table I.

TABLE I

SUPERCONDUCTING TAPE PROPERTIES

\begin{tabular}{ll}
\hline \hline Critical current, $I_{\mathrm{c}}$ [self-field, 77 K] & $100 \mathrm{~A}$ \\
$\mathrm{n}$-value, $n$ & 20 \\
Conductor width, $w$ & $4 \mathrm{~mm}$ \\
Superconducting layer thickness, $d_{\mathrm{sc}}$ & $1 \mu \mathrm{m}$ \\
Spacing between superconducting layers & $0.2 \mathrm{~mm}$ \\
Critical current density, YBCO layer, $J_{\mathrm{c} 0}$ & $2.5 \times 10^{10} \mathrm{~A} / \mathrm{m}^{2}$ \\
Characteristic electric field, $E_{0}$ & $1 \times 10^{-4} \mathrm{~V} / \mathrm{m}$ \\
\hline \hline
\end{tabular}

In addition to the standard analyses of the DC (critical current) and AC (AC loss) properties of the coil, the addition of flux diverters [5] is explored to improve the coil's performance. The flux diverters are represented by the same relative permeability for the strong magnetic material $\left(\mathrm{B}_{\text {sat }} \approx\right.$ $1.7 \mathrm{~T}, \mu_{\mathrm{r}, \max } \approx 12440$ at $75 \mathrm{~A} / \mathrm{m}$ ) used in [10]. Diverters of thickness $1 \mathrm{~mm}$ of this material are placed along both sides of the coil, as shown in Fig. 1, with a $1 \mathrm{~mm}$ gap representing the coil former and any insulation layer present.

To apply a current to the coil, pointwise constraints are applied to each $n$-th turn (i.e., each superconducting layer) of the coil by integrating $J_{\varphi}$ across each superconducting layer sub-domain such that $I_{\mathrm{n}}=\int J_{\varphi} \cdot d s_{\mathrm{n}}=I_{\text {app }}(t)$, where $s_{\mathrm{n}}$ is the cross-sectional area of each turn and $I_{\text {app }}$ is an appropriate function for the current waveform: a ramp function for the DC analysis, and a sinusoidal waveform for an AC current. Appropriate magnetic field boundary conditions are also set in the model: for a transport current only, $H_{\mathrm{r}}=H_{\mathrm{z}}=0$, for a sufficiently large air sub-domain, and for the in-field analysis (i.e., for an externally applied magnetic field), $H_{\mathrm{z}}=H_{\text {app }}, H_{\mathrm{r}}=$ 0 . In this paper, $H_{\text {app }}=H_{0}\left(t / t_{\text {ramp }}\right)$ for $t \leq t_{\text {ramp }}$, a ramp function that remains constant once the field reaches the magnitude of interest, which simulates a DC background field applied in the $z$ direction, perpendicular to the coil axis.

\section{Modelling Results}

\section{A. DC Analysis - Coil Critical Current}

In this section, an analysis of the DC properties of two coils - one of 20 turns, and another of 50 turns - is carried out for external fields $B=0,0.3$ and $0.6 \mathrm{~T}$, with and without flux diverters. Fig. 2 shows an example of how the field, and then current, is applied to the coil, for $B=0.3 \mathrm{~T}$ (ramped over $1 \mathrm{~s}$ ) and $I_{0}=110 \mathrm{~A}$ (ramped at $50 \mathrm{~A} / \mathrm{s}$ ). After ramping the field, there is a waiting period of $0.2 \mathrm{~s}$ to allow for flux relaxation [4] before ramping the current. The STOP function in Comsol is employed to terminate the simulation once a suitably large electric field is reached, which, in this case, was $E_{\text {coil }}=5 E_{0}$ (i.e., when the voltage developed across the coil terminals is five times the voltage when carrying the critical current, $I_{\mathrm{c}}$, as defined by the characteristic electric field $E_{0}$ ). This average electric field, $E_{\text {coil }}$, is calculated by integrating the local electric field [15], [16], as given by (2), where $r_{1}=r_{\mathrm{i}}=30$ $\mathrm{mm}$.

$$
E_{\text {coil }}=\sum_{n=1}^{N} \int 2 \pi r_{\mathrm{n}} E_{\varphi} \cdot d s_{n} / \sum_{n=1}^{N} 2 \pi r_{\mathrm{n}} \cdot s_{n}
$$

where $N$ is the total number of turns in the coil, $r_{\mathrm{n}}$ is the radius of each turn, and $E_{\varphi}$ is the local electric field.

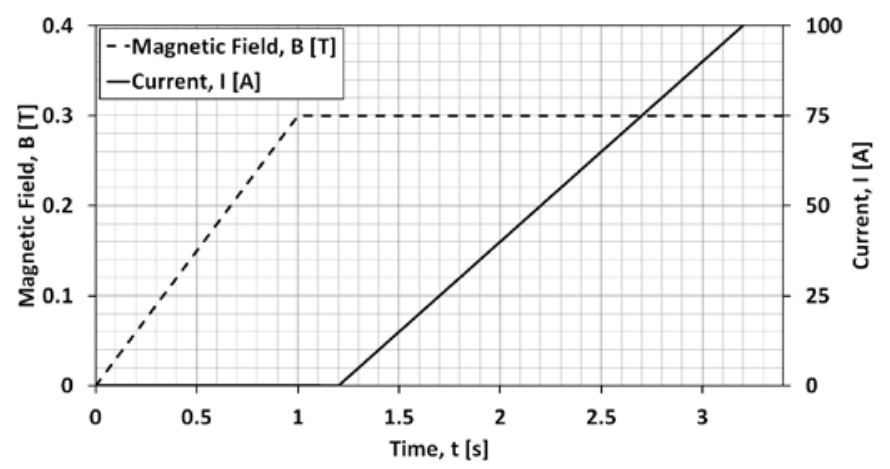

Fig. 2. Example applied external magnetic field and current for the DC analysis of coil performance.

Fig. 3 shows the $I-V$ curves for the 50 turn coil for external fields $B=0,0.3$ and $0.6 \mathrm{~T}$, with and without flux diverters, including the characteristic electric field for reference. Table II compares all of the calculated critical currents for both coils (20 and 50 turns) for all scenarios under analysis. From these results, it is clear that the presence of the external field has a reducing effect on the coil critical current. It is also observed that the flux diverter in this configuration does not appear to improve the $I_{\mathrm{c}}$ of the coil, in-field or otherwise, and in all cases acts somewhat to reduce performance.

In order to understand why this occurs, Fig. 4 plots the electric field for each turn of the 50 turn coil for a DC applied current, with no external magnetic field, such that $E_{\text {coil }}$, which is the average electric field between the coil terminals, equals the characteristic electric field, $E_{0}\left(1 \times 10^{-4} \mathrm{~V} / \mathrm{m}\right)$, i.e., when the critical current $I_{\mathrm{c}}$ is reached. This corresponds to $55.5 \mathrm{~A}$ for no diverters, and 48.2 A with diverters.

In circular pancake coils, where the superconducting properties do not vary between turns, the innermost turns see a comparatively higher local magnetic field [15], which reduces the local $J_{\mathrm{c}}$ in this region and determines the critical current of the whole coil. Therefore, investigating the local field seen by this inner turn is significant for the overall performance of the coil. It can be seen in Fig. 4 that the presence of the flux diverters increases the local magnetic field around the inner turns, which results in a higher electric field in these turns, but 


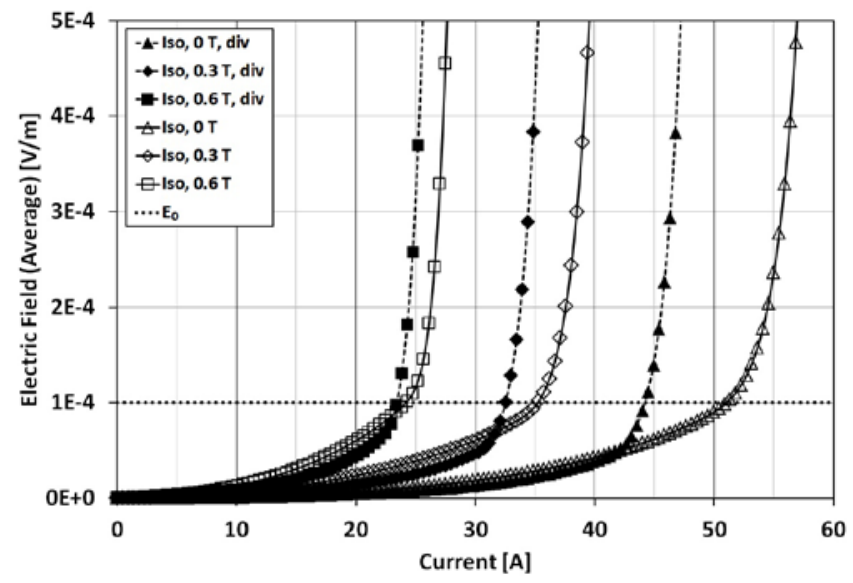

Fig. 3. I-V curves for the 50 turn coil.

TABLE II

COIL MOdel CRITICAL CURRENTS

\begin{tabular}{lccc}
\hline \hline MODEL & $\begin{array}{c}\text { FLUX } \\
\text { DIVERTER }\end{array}$ & $\begin{array}{c}\text { 20 TURN } \\
\text { COIL, } \mathbf{I}_{\mathrm{c}} \text { [A] }\end{array}$ & $\begin{array}{c}\text { 50 TURN } \\
\text { COIL, } \mathbf{I}_{\mathrm{c}} \text { [A] }\end{array}$ \\
\hline$J_{\mathrm{c}}(\mathrm{B}), B=0$ & NO & 64.5 & 55.5 \\
$J_{\mathrm{c}}(\mathrm{B}), B=0$ & YES & 62.5 & 48.2 \\
$J_{\mathrm{c}}(\mathrm{B}), B=0.3 \mathrm{~T}$ & NO & 40 & 38.5 \\
$J_{\mathrm{c}}(\mathrm{B}), B=0.3 \mathrm{~T}$ & YES & 38 & 35.5 \\
$J_{\mathrm{c}}(\mathrm{B}), B=0.6 \mathrm{~T}$ & NO & 27.5 & 26.5 \\
$J_{\mathrm{c}}(\mathrm{B}), B=0.6 \mathrm{~T}$ & YES & 26 & 25.5 \\
\hline \hline
\end{tabular}

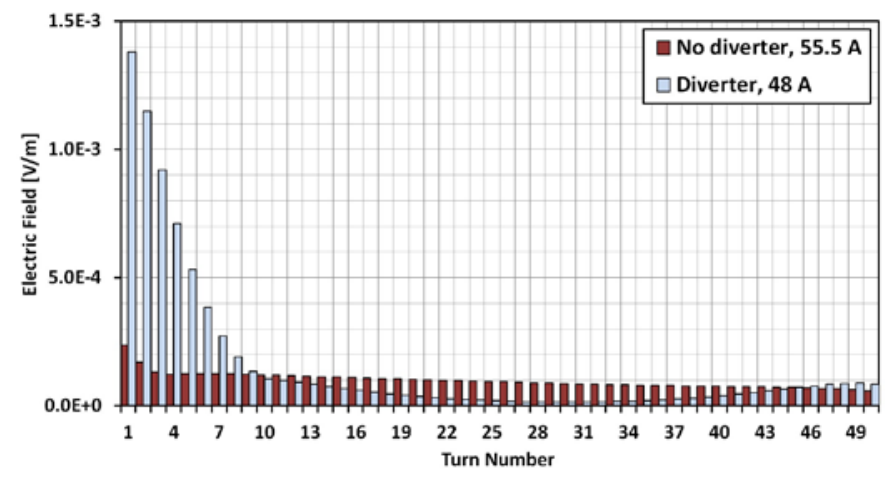

Fig. 4. Electric field for each turn of the 50 turn coil for a DC applied current, with no external magnetic field, such that $E_{\text {coil }}$, the average electric field between the coil terminals, equals the characteristic electric field, $E_{0}\left(1 \times 10^{-4}\right.$ $\mathrm{V} / \mathrm{m})$.

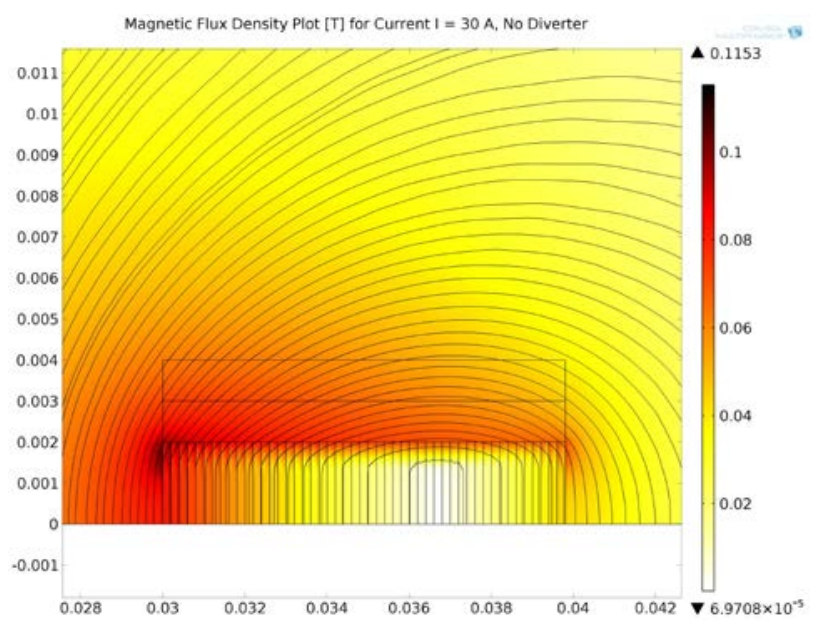

Fig. 5. Magnetic flux density plot for the 50 turn coil, without flux diverters, at $\mathrm{I}=30 \mathrm{~A}$ for a ramped current. conversely, there is a significantly reduced electric field (i.e., local magnetic field) in the central turns (in this case, approximately turns 10-40). Fig. 5 shows a plot of the magnetic flux density for the 50 turn coil, without flux diverters, at $\mathrm{I}=30$ A for a ramped current, and Fig. 6 shows this case with flux diverters. As described in [5], the diverters act to attract flux out of the coil in these regions, effecting a reduction in the AC loss, which is explored in the next section.

The results suggest a more detailed geometric optimization of the flux diverter is required to avoid an excessive increase in magnetic flux density at the coil edges, especially in the vicinity of the inner turn.

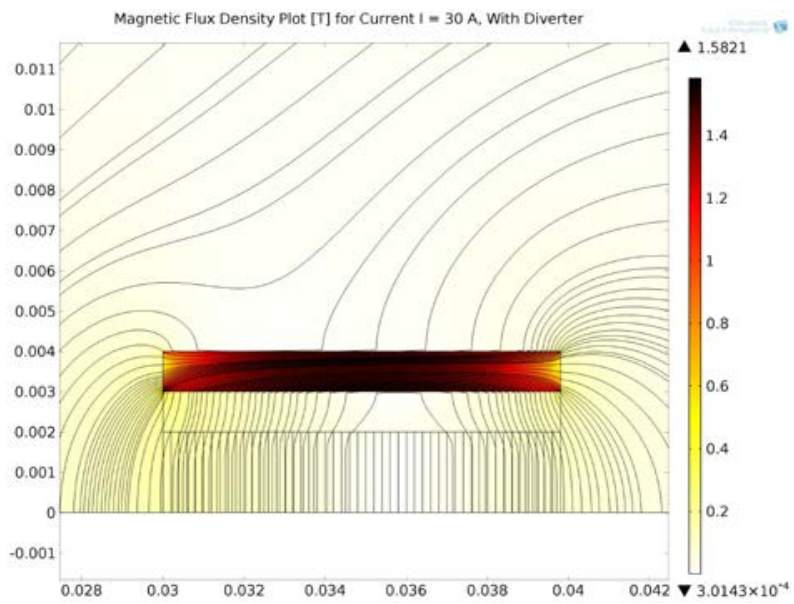

Fig. 6. Magnetic flux density plot for the 50 turn coil, with flux diverters, at I $=30 \mathrm{~A}$ for a ramped current.

\section{B. AC Analysis - Coil AC Loss}

In this section, an analysis of the AC properties of the two coils is carried out for external fields $B=0,0.3$ and $0.6 \mathrm{~T}$, with and without flux diverters, for a frequency of $50 \mathrm{~Hz}$. Fig. 7 shows an example of how the field, and then current, is applied to the coil, for $B=0.3 \mathrm{~T}$ (ramped over $1 \mathrm{~s}$ ) and $I_{0}=30$ A (of frequency $20 \mathrm{~Hz}$ as an example). As previously, after ramping the field, there is a waiting period of $0.2 \mathrm{~s}$ to allow for flux relaxation [4] before applying the AC current. The hysteretic AC loss in J/cycle in the superconducting layers, $Q_{\text {ac }}$, is calculated by the integrating the product of the local electric field and current density [16], as given by (3).

$$
Q_{\mathrm{ac}}=\int_{0}^{T} \sum_{n=1}^{N} \int 2 \pi r_{\mathrm{n}} E_{\varphi} \cdot J_{\varphi} d s_{n} d t
$$

where $T(1 / \mathrm{f})$ is the period, $E_{\varphi}$ is the local electric field, and $J_{\varphi}$ is the local critical current density.

The first half-cycle is usually considered a 'transient' and ignored as it has less dissipation because the superconductor is magnetized from its virgin state [17]. It is also common practice, in order to improve computation time, to integrate only the second half-cycle and double the calculated loss due to its hysteretic nature and symmetric waveform [18]. However, in complex electromagnetic situations such as these, where the external field can have additive and subtractive effects on the AC self-field, the AC loss should be calculated over at least 1.5 cycles, i.e., including the $2^{\text {nd }} / 3^{\text {rd }}$ half-cycles. 


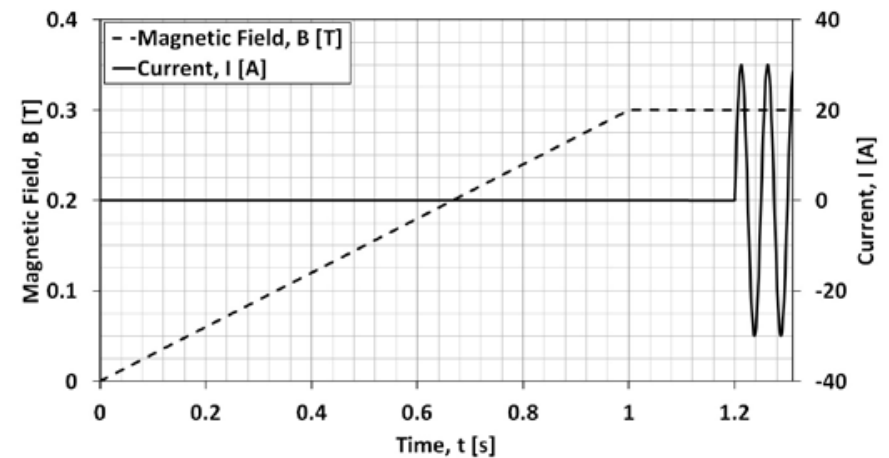

Fig. 7. Example applied external magnetic field and current for the AC analysis of coil performance.

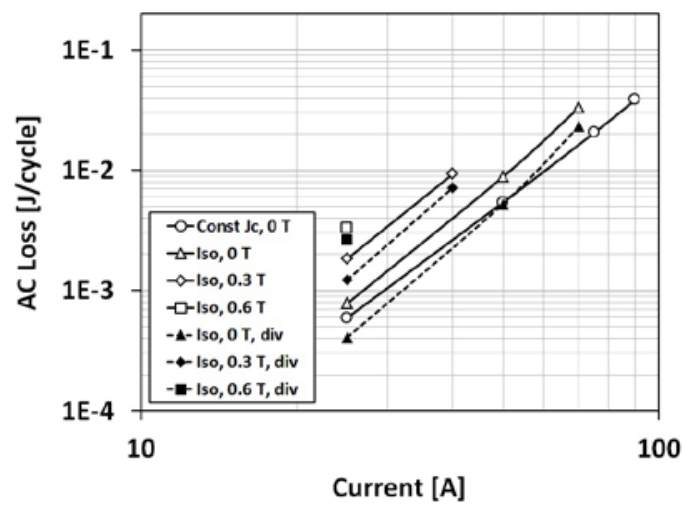

Fig. 8. AC loss calculations for the 20 turn coil, including a constant $J_{\mathrm{c}}$ case, as well as the isotropic $J_{\mathrm{c}}(B)$ model, with and without a flux diverter.

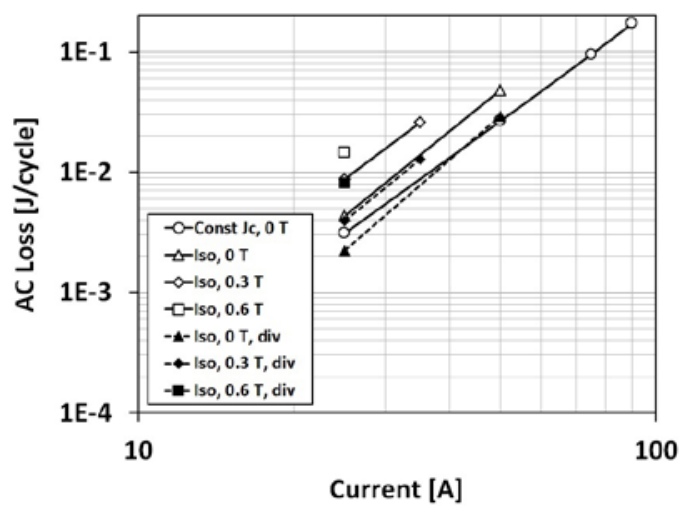

Fig. 9. AC loss calculations for the 50 turn coil, including a constant $J_{\text {c }}$ case, as well as the isotropic $J_{\mathrm{c}}(B)$ model, with and without a flux diverter.

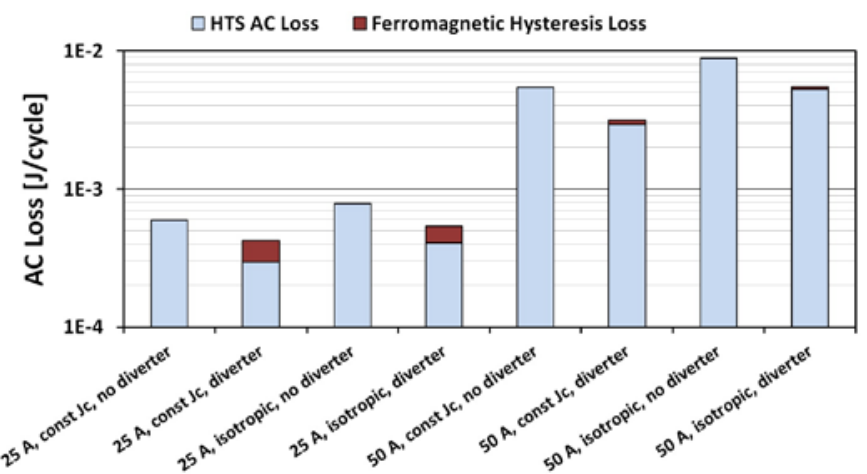

Fig. 10. AC loss including flux diverter hysteretic loss, no external field, for $I_{0} / I_{\mathrm{c}}=0.25,0.5$.
Fig. 8 shows a comparison of the raw AC loss calculations, i.e., not including the flux diverter hysteretic loss, for the isotropic $J_{\mathrm{c}}(B)$ models with external fields of $0,0.3$ and $0.6 \mathrm{~T}$, and with and without flux diverters, for the 20 turn coil. Fig. 9 shows the same comparison for the 50 turn coil. The result for a constant $J_{\mathrm{c}}$ for each coil is also provided as a base example case. This indicates the effect of a more realistic $J_{\mathrm{c}}$ on the AC loss, which increases as the magnitude of the external field is increased. For both the 20 and 50 turn coils, there is a clear decrease in the raw AC loss by using the diverters, even in the presence of an external field.

Fig. 10 shows the total AC loss for a number of cases, including the flux diverter's hysteretic loss, which is calculated by obtaining the peak magnetic field seen by the diverter as described in [9], but without an external field, for $I_{0} / I_{\mathrm{c}}=0.25$ and 0.5 . The AC loss is reduced with the use of the diverters, even when including the additional ferromagnetic hysteresis loss of the diverter, which is consistent with previous results for an infinitely long stack of superconducting tapes [5].

However, for the in-field cases, the diverter hysteretic loss calculation cannot be applied in the same way as [5], [9], [10], due to the presence of the DC background field and its interaction with the self-field from the AC transport current in the coil, resulting in asymmetric hysteresis loops experienced by the diverter. This requires more detailed experimental data on the material forming the flux diverter and/or a more complicated numerical model, such as [19]. Indeed, modelling such hysteresis loops in hybrid superconductor-ferromagnet structures is an area of research that needs some attention in the future.

In addition, since the effectiveness of a flux diverter is maximized when below the saturation limit/field of the material used, any external field will act to limit its benefit in reducing AC loss in the in-field case. The complex interaction between the current and magnetic field in relation to the flux diverter warrants further investigation.

\section{CONCLUSION}

In this paper, the in-field behaviour of high-temperature superconducting (HTS) coils was investigated using a twodimensional (2D) axisymmetric model of a circular pancake coil based on the H-formulation and implemented in Comsol Multiphysics 4.3a. The use of flux diverters to improve an HTS coil's performance with respect to its AC (AC loss) and DC (maximum allowable/critical current) characteristics, for various background magnetic fields, was also investigated. While the AC loss can be reduced using this technique, even when including the additional ferromagnetic hysteretic loss of the diverter, it can have a small detrimental effect on the DC properties. Further geometric optimization for the diverter, as well as consideration of the direction of applied field, is required. The next step in this research is to extend such a model to three dimensions (3D) in order to allow more complex electromagnetic situations to be modelled; in particular, applying external magnetic fields in directions other than perpendicular to the coil axis. 


\section{ACKNOWLEDGMENT}

Dr Mark Ainslie would like to acknowledge the support of a Royal Academy of Engineering Research Fellowship. Di Hu and Jin Zou would like to acknowledge support of Churchill College, Cambridge, the China Scholarship Council and the Cambridge Commonwealth, European and International Trust.

\section{REFERENCES}

[1] M. D. Ainslie et al., "Numerical analysis and finite element modelling of an HTS synchronous motor," Physica C, vol. 470, pp. 1752-1755, Nov. 2010.

[2] E. Pardo, J. Kovac, and J. Souc, "Power Loss in ReBCO Racetrack Coils Under AC Applied Magnetic Field and DC Current," IEEE Trans. Appl. Supercond., vol. 23, no. 3, pp. 4701305, Jun. 2013.

[3] H. W. Weijers et al., "High Field Magnets with HTS Conductors," IEEE Trans. Appl. Supercond., vol. 20, no. 3, Jun. 2010.

[4] Z. Hong et al., "AC Losses of Superconducting Racetrack Coil in Various Magnetic Conditions," IEEE Trans. Appl. Supercond., vol. 21, no. 3, pp. 2466-2469, Jun. 2011.

[5] M. D. Ainslie, W. Yuan, and T. J. Flack, "Numerical Analysis of AC Loss Reduction in HTS Superconducting Coils Using Magnetic Materials to Divert Flux,” IEEE Trans. Appl. Supercond., vol. 23, pp. 470014, Jun. 2013.

[6] COMSOL, Inc. www.comsol.com

[7] M. D. Ainslie, T. J. Flack, Z. Hong, and T. A. Coombs, "Comparison of first- and second-order 2D finite element models for calculating AC loss in high temperature superconductor coated conductors," Intl. J. Comput. Math. Electr. Electron. Eng., vol. 30, no. 2, pp. 762-744, 2011.

[8] M. D. Ainslie et al., "Modeling and Electrical Measurement of Transport AC Loss in HTS-Based Superconducting Coils for Electric Machines," IEEE Trans. Appl. Supercond., vol. 21, no. 3, pp. 32653268, 2011.

[9] M. D. Ainslie et al., "An improved FEM model for computing transport AC loss in coils made of RABiTS YBCO coated conductors for electric machines,” Supercond. Sci. Technol., vol. 24, no. 4, pp. 045005, 2011.

[10] M. D. Ainslie, T. J. Flack, and A. M. Campbell, "Calculating transport AC losses in stacks of high temperature superconductor coated conductors with magnetic substrates using FEM," Physica C, vol. 472, pp. 50-56, 2012.

[11] C. J. G. Plummer, and J. E. Evetts, "Dependence of the Shape of the Resistive Transition on Composite Homogeneity in Multifilamentary Wires,” IEEE Trans. Magn., vol. 23, no. 2, pp. 1179-1182, Mar. 1987.

[12] J. Rhyner, "Magnetic properties and AC-losses of superconductors with power law current-voltage characteristics," Physica C, vol. 212, pp. 292-300, Jul. 1993.

[13] Y. B. Kim, C. F. Hempstead, and A. R Strnad, "Critical persistent currents in hard superconductors,” Phys. Rev. Lett., vol. 9, pp. 306-309, 1963.

[14] SuperPower, Inc. www.superpower-inc.com

[15] M. Zhang et al., "Study of second generation, high-temperature superconducting coils: Determination of critical current," J. Appl. Phys., vol. 111, pp. 083092, 2012.

[16] F. Gömöry et al., "AC Loss in Pancake Coil Made From 12 mm Wide REBCO Tape”, IEEE Trans. Appl. Supercond., vol. 23, no. 3, pp. 5900406, Jun. 2013.

[17] F. Grilli et al., "Self-Field Effects and AC Losses in Pancake Coils Assembled From Coated Conductor Roebel Cables,” IEEE Trans. Appl. Supercond., vol. 24, no. 3, Jun. 2014.

[18] P. Kruger, "Optimisation of hysteretic losses in high-temperature superconducting wires," $\mathrm{PhD}$ thesis, Karlsruher Institute für Technologie (KIT), Karlsruhe, Germany, 2014.

[19] Z. Zhao et al., "Modeling Magnetic Hysteresis Under DC-Biased Magnetization Using the Neural Network,” IEEE Trans. Magn., vol. 45, no. 10, pp. 3958-3961, Oct. 2009. 